\title{
Capturas ícticas incidentales de la pesca ornamental en el período de aguas bajas en el área de influencia de Puerto Carreño, Orinoquia colombiana
}

\author{
Saúl Prada-Pedreros ${ }^{1}$, Jhon González-Forero ${ }^{2}$, Juan Mondragón-Estupiñan ${ }^{2}$ \\ ${ }^{1}$ Departamento de Biología. Facultad de Ciencias. Pontificia Universidad Javeriana. Bogotá D.C., Colombia, \\ Carrera 7 No. 40 - 62 Tel: (571) 3208320 \\ ${ }^{2}$ Proyecto Curricular De Licenciatura en Biología. Facultad de Ciencias y Educación. Universidad Distrital Francisco José de Caldas. Bogotá D.C., \\ Colombia, Carrera 3 N. 26 a 40 Tel (571) 2842801 \\ *saul.prada@gmail.com; enriquemime@gmail.com; jucamon84@gmail.com
}

Recibido: 03-12-2009; Aceptado: 05-02-2010

\begin{abstract}
Resumen
Objetivo. El presente estudio evalúa las capturas ícticas incidentales de la pesca de especies ornamentales, en términos de abundancia y riqueza, en la zona de influencia del municipio de Puerto Carreño, Vichada, Colombia. Materiales y métodos. Para esto, se realizaron muestreos en los ríos Bita, Orinoco y en Caño Negro, durante el período de aguas bajas, en marzo y abril de 2007. A partir del acompañamiento realizado a las jornadas de pesca ornamental, se colectaron 152 especies entre incidentales y objeto. Se estimó la captura por unidad de esfuerzo (CPUE) y la composición porcentual de las capturas ícticas de las especies objeto versus las incidentales. Resultados. Los valores más altos de CPUE para especies objeto corresponden a los estimados para: Dicrossus maculatus, Hemigrammus rodhostomus, Corydoras cf. melanistius, Peckoltia sp. 2. y Hemiancistrus sp.; Por otro lado, los valores más altos para las especies ícticas incidentales corresponden a: Hemigrammus sp., Moenkhausia sp., Hemigrammus levis, Aphanothorulus cf. ammophilus, Triportheus angulatus y Moenkhausia lepidura. El porcentaje en riqueza para las especies ícticas incidentales varió entre el $92 \%$ y $98 \%$. No obstante, el porcentaje en abundancia de las especies objeto fue mayor que el obtenido para las capturas incidentales variando entre $73 \%$ y $83 \%$, con excepción de la obtenida en la pesca en los afloramientos rocosos del río Orinoco que fue del 17\%. Conclusiones. El impacto de la pesca ornamental sobre las especies de peces capturadas incidentalmente se observa principalmente a nivel de riqueza, pero no a nivel de abundancia.
\end{abstract}

Palabras clave: pesca ornamental, capturas incidentales, CPUE, Puerto Carreño

\begin{abstract}
Incidental bycatch by ornamental fishing during the low-water season in the influence zone of Puerto Carreño, Colombian Orinoco Basin. Objective. The present study assesses the incidental fish catches in the fishing of ornamental species in terms of richness and abundance within the influence zone of Puerto Carreño, Vichada, Colombia. Materials and methods. Samplings were carried out in the Orinoco River and Bita River and Caño Negro stream during the low-water season, in March and April of 2007. Target and incidental species collected during the ornamental fishing totalized 152. Catches per unit of effort (CPUE) as well as the percentage composition of fish catches of target vs. incidental species were estimated. Results. The highest values of CPUE of fishes with ornamental value correspond to: Dicrossus maculatus, Hemigrammus rodhostomus, Corydoras cf. melanistius, Peckoltia sp. 2 and Hemiancistrus sp., and the highest values of incidental species were for: Hemigrammus sp., Moenkhausia sp., Hemigrammus levis, Aphanothorulus cf. ammophilus, Triportheus sp. y Moenkhausia lepidura. The richness percentage of incidental species varied between $92 \%$ and $98 \%$. However the abundance percentage of target species was always higher than the percentage of incidental catches varying between $73 \%$ and $83 \%$ with the exception of that obtained in the fisheries of the Orinoco River outcrops that was $17 \%$. Conclusions. The impact of the fishing ornamental fish on species taught incidentally is seen mainly at the level of richness, but not at the level of abundance.
\end{abstract}

Key words: bycatch, CPUE, ornamental fish, Puerto Carreño 


\begin{abstract}
Resumo
Capturas ícticas incidentais da pesca ornamental no período de níveis de água baixo na área de influencia de Puerto Carreño, Orinoquia colombiana. Objetivo. Este estudo avalia a captura incidental de peixes produto da pesca ornamental, em termos de abundância e riqueza, na bacia hidrográfica do município de Puerto Carreño, Vichada, Colômbia. Materiais e métodos. Para isso, realizaram-se amostragens nos rios Bita, Orinoco e Caño Negro, durante o período de níveis da água baixos, em Março e Abril de 2007. A partir do acompanhamento direto feito na pescaria ornamental, foram coletadas 152 espécies entre as alvo e as incidentais. Estimaram-se as capturas por unidade de esforço (CPUE) e a composição percentual das capturas das espécies-alvo versus as incidentais. Resultados. Os maiores valores de CPUE para as espécies-alvo correspondem aos estimados para: Dicrossus maculatus, Hemigrammus rodhostomus, Corydoras cf. melanistius, Peckoltia sp. 2 e Hemiancistrus sp., e os maiores valores para espécies incidentais correspondem a: Hemigrammus sp., Moenkhausia sp., Hemigrammus levis, Aphanothorulus cf. ammophilus, Triportheus angulatus e Moenkhausia lepidura. O percentual da riqueza das espécies de peixes incidentais variou entre $92 \%$ e $98 \%$. No entanto, o percentual da abundância das espéciesalvo foi maior que aquele pra as espécies incidentais variando entre $73 \%$ e $83 \%$, com exceção da obtida na pesca nos afloramentos rochosos do río Orinoco o qual foi de $17 \%$. Conclusões. O impacto da pesca ornamental sobre as espécies de peixes capturadas incidentalmente pode ser observado principalmente ao nível de riqueza, mas não ao nível de abundância.
\end{abstract}

Palavras-chave: pesca ornamental, Captura incidental, CPUE, Puerto Carreño

\section{Introducción}

La extracción y comercialización de peces ornamentales es una actividad que se ha desarrollado en el país por más de 50 años (1), constituyendo una fuente importante de ingresos en zonas alejadas de los centros urbanos (2) y para algunas comunidades es la única actividad económica que presentan (1). Colombia se ubica dentro de los 15 principales países exportadores; durante los últimos cinco años sus exportaciones se encuentran alrededor de los 20 millones de individuos (3). El $88 \%$ de los peces ornamentales comercializados en Colombia son extraídos de la región de la Orinoquia, el 10\% de la Amazonia, el 1,7\% de la región Pacifica y el 0,3\% de la cuenca del Magdalena (3). En la región de la Orinoquia, Puerto Carreño aporta cerca del 7\% de esta producción (1). Los peces de interés comercial son extraídos de su ambiente natural, y si bien se tiene información acerca del número de individuos por especie movilizados, lo mismo no ocurre con información biológica y ecológica del recurso (3). Otro gran vacío de información es la carencia de datos sobre las capturas ícticas incidentales durante la pesca de peces ornamentales. Así, el presente estudio tiene por objeto evaluar las capturas ícticas incidentales de la pesca ornamental, en términos de abundancia y riqueza, en el municipio de Puerto Carreño, Vichada, Colombia.

\section{Materiales y métodos}

Puerto Carreño se localiza a $6^{\circ} 11^{\prime}$ latitud norte, $67^{\circ} 29^{\prime}$ longitud oeste y a $50 \mathrm{msnm}$. Su temperatura anual media es de $28,1^{\circ} \mathrm{C}$, con valores que oscilan entre 18 y $42,8^{\circ} \mathrm{C}$; el brillo solar es de 2.253 horas al año; la precipitación anual es de $2.174 \mathrm{~mm}$; la evaporación media anual es de $2.041 \mathrm{~mm}$; la humedad relativa del $70 \%$ varía entre $49 \%$ y $88 \%$ (4).
Los muestreos se llevaron a cabo en diferentes cuerpos de agua en la zona de influencia de Puerto Carreño en el departamento de Vichada (río Bita, río Orinoco y caño Negro) de donde se extraen los peces ornamentales (Figura 1). Para llevar a cabo el registro y captura de las especies ícticas ornamentales e incidentales, se efectuaron muestreos directos acompañando a los pescadores durante sus faenas de pesca. El arte de pesca utilizado con mayor frecuencia fue la red de arrastre o "chinchorro" y en algunos casos se utilizó el rayero y la nasa. Las dimensiones de las redes de arrastre fueron diferentes para cada uno de los ríos, caños y afluentes como se describen a continuación. Para el río Bita fue usada una red de arrastre de $8 \mathrm{~m}$ de longitud por $1,50 \mathrm{~m}$ de altura; en este río también se utilizó la nasa. Esta última se utiliza para la pesca de la arawana (Osteoglossum ferreirae) y consiste en un soporte metálico de $70 \mathrm{~cm}$ de ancho y $40 \mathrm{~cm}$ de largo. Adherida a este, se dispone una malla de ojo de $1 \mathrm{~mm}$ en forma de bolsa, que alcanza una profundidad de $40 \mathrm{~cm}$.; el soporte se sujeta por uno de sus lados a una base de madera maciza. En el caño Negro se utilizó una red de arrastre de $6 \mathrm{~m}$ de longitud por 1,40 $\mathrm{m}$ de altura y el rayero para la pesca de rayas (Potamotrygon motoro). El rayero consiste en un eje de 1,20 m de altura, en uno de los extremos se encuentra un recipiente redondo en el que hay una abertura que conduce a la raya a una red en forma de bolsa de $40 \mathrm{~cm}$, el rayero se coloca encima de la raya y esta se conduce a la bolsa de donde es retirada y dejada en el bote para el transporte. En el río Orinoco la red de arrastre empleada fue de $6,8 \mathrm{~m}$ de longitud por 1,50 $\mathrm{m}$ de altura. La técnica utilizada en todas las faenas de pesca, consistió en múltiples lances. Un lance de chinchorro consiste en arrastrar la red hacia la orilla con el fin de encerrar y capturar las especies objeto. El lance con la nasa o con el rayero se realiza una vez el pescador detecta visualmente al pez. El uso de otras técnicas de pesca como el Zangarreo (1), no fue utilizado durante las faenas. 
Durante la pesca de las especies objeto, se colectaron todos los individuos de las especies capturadas incidentalmente, registrando el número de individuos de las especies objeto y seleccionando algunos especímenes para su posterior identificación en el laboratorio. Los individuos colectados, fueron fijados en campo en formol al $10 \%$ y empacados en bolsas de cierre hermético y recipientes de plástico con su respectiva etiqueta. En cada uno de los registros, se indicó el arte y el tiempo empleados en la pesca y se tomaron fotografías de los individuos lo que permitió registrar los patrones de coloración en fresco para algunas especies. Posteriormente, los ejemplares colectados fueron lavados con abundante agua para luego ser preservados en alcohol al 70\%. La identificación de las especies fue llevada a cabo en el Laboratorio de Ictiología de la Pontificia Universidad Javeriana, Bogotá. Las especies fueron identificadas mediante el uso de bibliografía y claves especializadas. Los ejemplares debidamente identificados y etiquetados se depositaron en La Colección de Peces del Museo Javeriano de Historia Natural, Lorenzo Uribe, S.J. bajo el acrónimo de MPUJ.

La riqueza se calculó mediante el conteo de especies. La abundancia relativa de las especies se estimó para cada punto de muestreo a partir de la captura por unidad de esfuerzo (CPUE) en términos de número de individuos por especie/minutos de pesca. El esfuerzo fue estimado a partir del tiempo efectivo de pesca. El tiempo efectivo de pesca está constituido por: la suma del tiempo en los lugares de pesca buscando los peces, el tiempo en que el arte está funcionando y el tiempo de manipulación de la colecta en minutos (5), como se indica en la ecuación:

$$
n_{i}=c_{i} / t
$$

En donde $n_{i}$ es la abundancia relativa de la especie $i, c_{i}$ la cantidad de individuos recolectados de esa especie y t el tiempo en minutos de cada una de las jornadas (6).

Para estimar la abundancia relativa de las especies en cada sitio de extracción, fueron organizados los datos en matrices en hojas de cálculo Excel. También se estimó la frecuencia de ocurrencia para cada una de las especies, considerando el número de apariciones de cada especie en función del número total de lances por sitio.

\section{Resultados}

\section{Riqueza}

La riqueza total fue de 152 especies pertenecientes a 8 ordenes, este valor corresponde tanto a especies ícticas
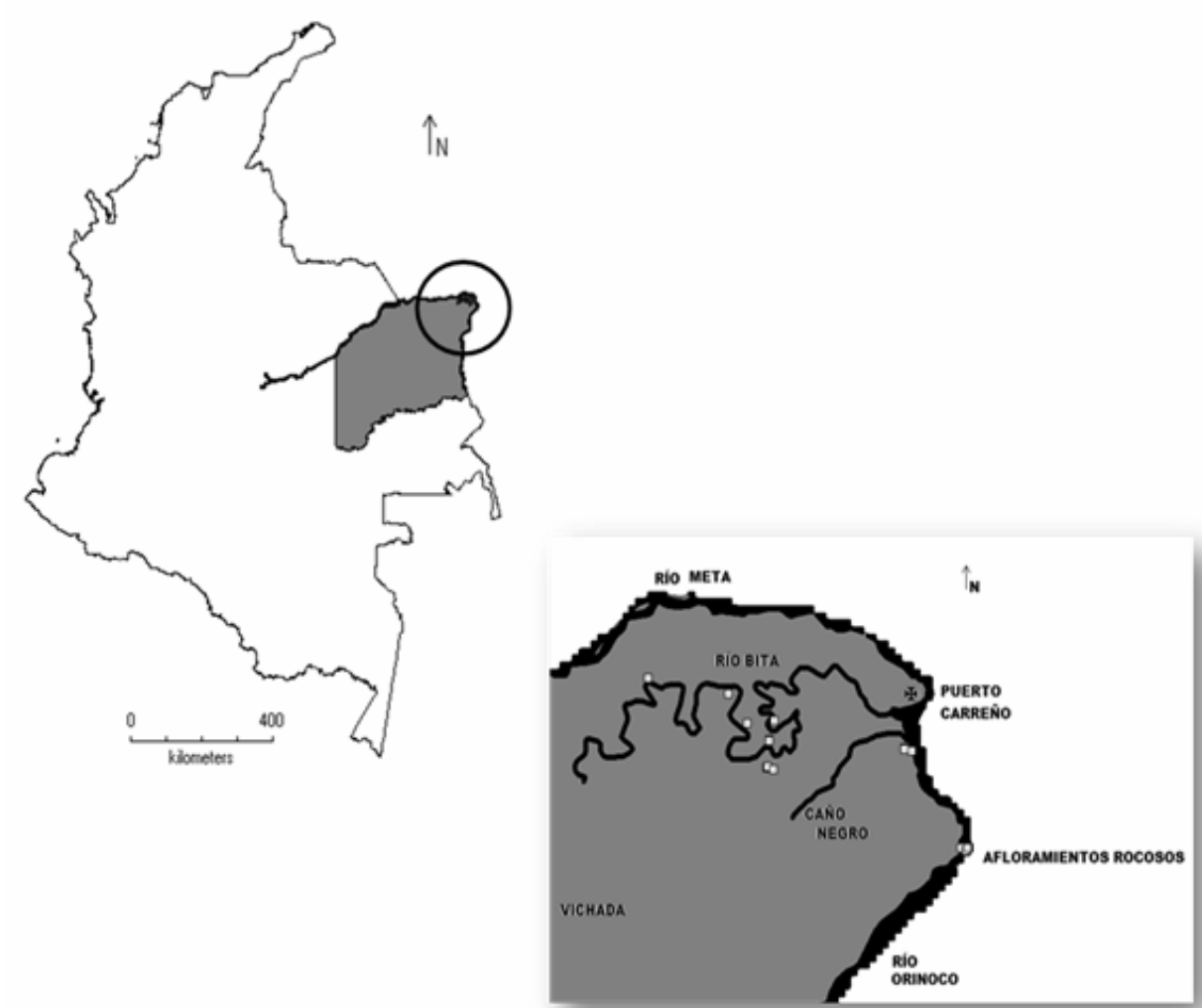

Figura 1. Ubicación geográfica de los sitios de muestreo. 
ornamentales como a las ícticas incidentales. El orden que presenta un mayor número de especies es Characiformes con 74 que equivale al $49 \%$, seguido por Siluriformes con 47 especies (31\%), el orden Perciformes presentó 23 especies (15\%), Gymnotiformes 3 (2\%) y de Rajiformes 2 (1\%); los ordenes Osteoglossiformes, Clupeiformes y Beloniformes presentan el menor número de especies con solo una, estos tres órdenes solo representan el $2 \%$ del total de especies. (Figura 2).

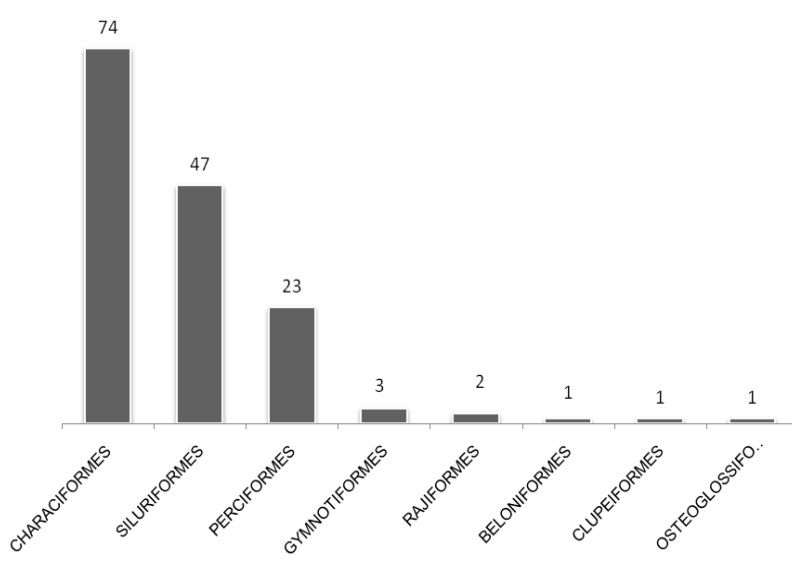

Figura 2. Número de especies por cada orden.

Para los Characiformes la familia con mayor número de especies es Characidae, con 45, seguida por Anostomidae con 8 y Curimatidae con 5; mientras que, Prochilodontidae, Ctenoluciidae, Erythrinidae, Gasteropelicidae y Hemiodontidae presentaron 2 especies; Parodontidae, Chilodontidae, Crenuchidae, Acestrorhynchidae, Cynodontidae y Lebiasinidae presentan los valores más bajos con una especie (Figura 3).

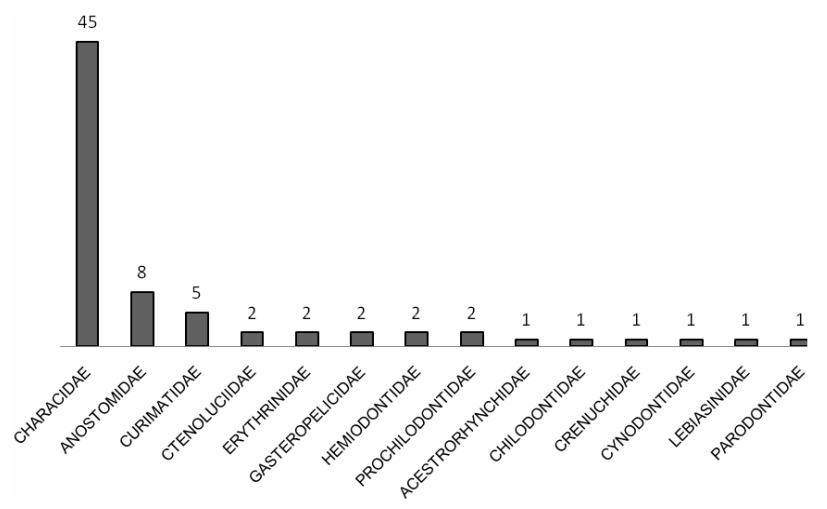

Figura 3. Número de especies de cada familia del orden Characiformes. Número total de especies: 74 .
En el orden Siluriformes se observa que la familia Loricariidae es la que mayor número de especies presenta, con 26 (55\%), le sigue Heptapteridae con 8 (17\%), Doradidae con $4(9 \%)$, mientras que Trichomycteridae presenta 3 especies (7\%); Pimelodidae junto con Callichthyidae registran un total de 2 (4\%) cada una y, las Pseudopimelodidae y Auchenipteridae, presentan una especie cada una, que corresponden al 2\% (Figura 4).

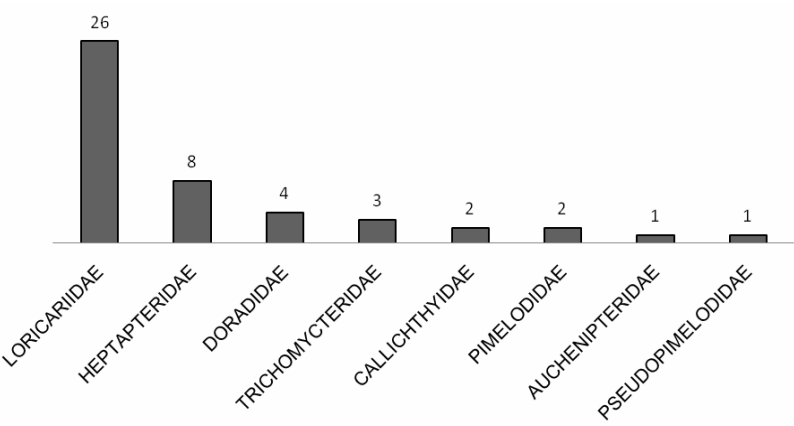

Figura 4. Número de especies de cada familia del orden Siluriformes. Número total de especies: 47.

Del orden Perciformes se colectaron 3 familias, de las cuales Cichlidae es la que presenta el mayor número de especies con $20(87 \%)$, Sciaenidae presenta dos (9\%) y Eleotridae presenta una especie (4\%).

\section{Abundancia relativa, frecuencia y porcentaje de especies objeto vs. incidentales}

\section{Río Bita}

En la jornada de pesca llevada a cabo en el río Bita, las especies objeto fueron tres: Dicrossus maculatus, Hemigrammus rodhostomus y Boulengerella lateristriga. Dicrossus maculatus presentó los valores de abundancia relativa $(20,35)$ y frecuencia $(\mathrm{F}=10)$ más altos. Le sigue Hemigrammus rodhostomus con 13,71 y 8 de abundancia y frecuencia, respectivamente. Por otro lado, Boulengerella lateristriga obtuvo un valor bajo de abundancia relativa $(1,08)$ y frecuencia $(\mathrm{F}=3)$. Dentro de las capturas incidentales se observó que las especies del genero Hemigrammus y Moenkhausia fueron las más abundantes y frecuentes (Tabla 1).

De las 39 especies capturadas en el río Bita, 36 (92\%) correspondieron a incidentales. En relación con los valores de abundancia, las especies objeto presentan un porcentaje del $73 \%$ (Tabla 2). 
Tabla 1. Valores de abundancia relativa en términos de CPUE $\left(n_{i}=c_{i} / t\right)$, porcentaje $(\%)$, frecuencia (F) y número de individuos $(\mathrm{N})$, para las especies objeto e incidentales, colectadas en el río Bita.

\begin{tabular}{|c|c|c|c|c|c|}
\hline \multicolumn{2}{|c|}{ ESPECIES } & \multirow{2}{*}{$\begin{array}{c}\text { Abundancia } \\
20,35\end{array}$} & \multirow{2}{*}{$\frac{\%}{42,53}$} & \multirow{2}{*}{$\frac{\text { F (Máxima }=10)}{10}$} & \multirow{2}{*}{$\frac{\mathbf{N}}{1526}$} \\
\hline 1 & Dicrossus maculatus* & & & & \\
\hline 2 & Hemigrammus rodhostomus* & 13,71 & 28,65 & 8 & 1028 \\
\hline 3 & Hemigrammus sp. & 2,23 & 4,66 & 8 & 167 \\
\hline 4 & Moenkhausia sp. & 2,15 & 4,49 & 7 & 161 \\
\hline 5 & Bryconamericus sp. & 2,11 & 4,41 & 2 & 158 \\
\hline 6 & Carnegiella marthae & 2,01 & 4,20 & 1 & 151 \\
\hline 7 & Boulengerella lateristriga* & 1,08 & 2,26 & 3 & 81 \\
\hline 8 & Biotodoma wavrini & 0,65 & 1,36 & 3 & 49 \\
\hline 9 & Brycon cf. melanopterus & 0,45 & 0,94 & 7 & 34 \\
\hline 10 & Nannostomus eques & 0,37 & 0,77 & 2 & 28 \\
\hline 11 & Satanoperca daemon & 0,37 & 0,77 & 2 & 28 \\
\hline 12 & Moenkhausia collettii & 0,35 & 0,73 & 4 & 26 \\
\hline 13 & Chalceus macrolepidotus & 0,27 & 0,56 & 4 & 20 \\
\hline 14 & Curimatella inmaculata & 0,19 & 0,40 & 1 & 14 \\
\hline 15 & Iguanodectes spilurus & 0,16 & 0,33 & 2 & 12 \\
\hline 16 & Acestrorhynchus microlepis & 0,15 & 0,31 & 5 & 11 \\
\hline 17 & Acanthodoras cataphractus & 0,13 & 0,27 & 2 & 10 \\
\hline 18 & Laemolyta taeniata & 0,13 & 0,27 & 2 & 10 \\
\hline 19 & Hemigrammus iota & 0,12 & 0,25 & 1 & 9 \\
\hline 20 & Hemiodon semitaeniatus & 0,11 & 0,23 & 2 & 8 \\
\hline 21 & Uaru fernandezyepezi & 0,09 & 0,19 & 2 & 7 \\
\hline 22 & Cyphocharax spilurus & 0,08 & 0,17 & 1 & 6 \\
\hline 23 & Mesonauta egregius & 0,08 & 0,17 & 1 & 6 \\
\hline 24 & Curimatella dorsalis & 0,07 & 0,15 & 3 & 5 \\
\hline 25 & Leporinus agassizii & 0,07 & 0,15 & 1 & 5 \\
\hline 26 & Hemigrammus elegans & 0,05 & 0,10 & 1 & 4 \\
\hline 27 & Moenkhausia lepidura & 0,05 & 0,10 & 2 & 4 \\
\hline 28 & Satanoperca juropari & 0,05 & 0,10 & 2 & 4 \\
\hline 29 & Crenicichla geayi & 0,04 & 0,08 & 3 & 3 \\
\hline 30 & Dekeyseria scaphirhyncha & 0,03 & 0,06 & 2 & 2 \\
\hline 31 & Moenkhausia oligolepis & 0,03 & 0,06 & 2 & 2 \\
\hline 32 & Rineloricaria formosa & 0,03 & 0,06 & 1 & 2 \\
\hline 33 & Satanoperca sp. & 0,03 & 0,06 & 2 & 2 \\
\hline 34 & Bryconops alburnoides & 0,01 & 0,02 & 1 & 1 \\
\hline 35 & Caquetaia sp. & 0,01 & 0,02 & 1 & 1 \\
\hline 36 & Characidium sp. & 0,01 & 0,02 & 1 & 1 \\
\hline 37 & Hyphessobrycon sp. & 0,01 & 0,02 & 1 & 1 \\
\hline 38 & Iguanodectes cf. adujai & 0,01 & 0,02 & 1 & 1 \\
\hline 39 & Oxyropsis acutirostra & 0,01 & 0,02 & 1 & 1 \\
\hline
\end{tabular}

Las especies destacadas con asterisco corresponden a las "especies objeto".

La frecuencia máxima hace referencia al número de lances por sitio. 
Tabla 2. Número $(\mathrm{N})$, porcentaje (valores entre paréntesis) y abundancia relativa (\%) de especies objeto e incidentales por lugar en cada jornada de pesca

\begin{tabular}{ccccc}
\hline & \multicolumn{2}{c}{ Especies objeto } & \multicolumn{2}{c}{ Especies incidentales } \\
\hline Lugar & N & \% & N & $\%$ \\
\hline Río Bita & $3(8)$ & 73 & $36(92)$ & 27 \\
Caño Negro & $3(8)$ & 82 & $33(92)$ & 18 \\
Río Orinoco & & & & \\
Afloramientos Rocosos & $2(2)$ & 17 & $80(98)$ & 83 \\
Pozo Bisal & $1(4)$ & 83 & $27(96)$ & 17 \\
\hline
\end{tabular}

\section{Caño Negro}

En la jornada de pesca llevada a cabo en caño Negro las especies objeto fueron tres: Corydoras cf. melanistius, Dicrossus maculatus y Potamotrygon motoro. Corydoras cf. melanistius presentó los valores de abundancia relativa $(5,44)$ y frecuencia $(8)$ más altos. Le sigue Dicrossus maculatus con 4,76 y 8 de abundancia y frecuencia, respectivamente. Por otro lado, Potamotrygon motoro obtuvo un valor muy bajo de abundancia relativa $(0,06)$ pero su frecuencia fue alta, (8). Dentro de las capturas incidentales se observó que las especies del genero Hemigrammus fueron las más abundantes (Tabla 3).

Tabla 3. Valores de abundancia relativa en términos de CPUE $\left(n_{i}=c / t\right)$, porcentaje $(\%)$, frecuencia $(F)$ y número de individuos (N), para las especies objeto e incidentales, colectadas en caño Negro.

\begin{tabular}{|c|c|c|c|c|c|}
\hline \multicolumn{2}{|c|}{ ESPECIE } & \multirow{2}{*}{$\begin{array}{c}\text { Abundancia } \\
5,44\end{array}$} & \multirow{2}{*}{$\frac{\%}{43,49}$} & \multirow{2}{*}{$\frac{\mathbf{F}(\text { Máxima }=\mathbf{8})}{8}$} & \multirow{2}{*}{$\frac{\mathbf{N}}{408}$} \\
\hline 1 & Corydoras cf. melanistius* & & & & \\
\hline 2 & Dicrossus maculatus* & 4,76 & 38,05 & 8 & 1286 \\
\hline 3 & Hemigrammus levis & 0,44 & 3,52 & 5 & 120 \\
\hline 4 & Hemigrammus cf. analis & 0,31 & 2,48 & 4 & 60 \\
\hline 5 & Nannostomus eques & 0,26 & 2,08 & 4 & 50 \\
\hline 6 & Moenkhausia collettii & 0,22 & 1,76 & 3 & 42 \\
\hline 7 & Crenicichla sp. 3 & 0,16 & 1,28 & 1 & 12 \\
\hline 8 & Apistogramma brevis & 0,09 & 0,72 & 3 & 23 \\
\hline 9 & Curimatella dorsalis & 0,09 & 0,72 & 3 & 18 \\
\hline 10 & Hemigrammus sp. & 0,09 & 0,72 & 2 & 17 \\
\hline 11 & Moenkhausia sp. & 0,09 & 0,72 & 1 & 18 \\
\hline 12 & Bryconops caudomaculatus & 0,07 & 0,56 & 1 & 13 \\
\hline 13 & Potamotrygon motoro* & 0,06 & 0,48 & 8 & 11 \\
\hline 14 & Microphilypnus ternetzi & 0,05 & 0,40 & 2 & 9 \\
\hline 15 & Moenkhausia lepidura & 0,04 & 0,32 & 2 & 7 \\
\hline 16 & Asterophisus batrachus & 0,03 & 0,24 & 1 & 2 \\
\hline 17 & Bryconops cf. giacopinii & 0,03 & 0,24 & 1 & 5 \\
\hline 18 & Characidium sp. & 0,03 & 0,24 & 1 & 6 \\
\hline 19 & Mesonauta egregius & 0,03 & 0,24 & 2 & 5 \\
\hline 20 & Microshemobrycon casiquiare & 0,03 & 0,24 & 1 & 5 \\
\hline 21 & Brycon cf. melanopterus & 0,02 & 0,16 & 2 & 4 \\
\hline
\end{tabular}




\begin{tabular}{|c|c|c|c|c|c|}
\hline \multicolumn{2}{|c|}{ ESPECIE } & \multirow{2}{*}{$\begin{array}{c}\text { Abundancia } \\
0,02\end{array}$} & \multirow{2}{*}{$\frac{\%}{0,16}$} & \multirow{2}{*}{$\frac{\mathbf{F}(\text { Máxima = 8) }}{1}$} & \multirow{2}{*}{$\begin{array}{l}\mathbf{N} \\
4\end{array}$} \\
\hline 22 & Hemigrammus iota & & & & \\
\hline 23 & Satanoperca daemon & 0,02 & 0,16 & 1 & 3 \\
\hline 24 & Acestrorhynchus microlepis & 0,01 & 0,08 & 1 & 1 \\
\hline 25 & Aequidens sp. & 0,01 & 0,08 & 2 & 2 \\
\hline 26 & Boulengerella lateristriga & 0,01 & 0,08 & 1 & 1 \\
\hline 27 & Chalceus macrolepidotus & 0,01 & 0,08 & 1 & 1 \\
\hline 28 & Crenicichla sp. 2 & 0,01 & 0,08 & 1 & 1 \\
\hline 29 & Curimatopsis sp. & 0,01 & 0,08 & 1 & 1 \\
\hline 30 & Hemigrammus microstomus & 0,01 & 0,08 & 1 & 1 \\
\hline 31 & Hemigrammus schmardae & 0,01 & 0,08 & 1 & 2 \\
\hline 32 & Hyphessobrycon sweglesi & 0,01 & 0,08 & 1 & 2 \\
\hline 33 & Moenkhausia oligolepis & 0,01 & 0,08 & 1 & 1 \\
\hline 34 & Physopyroxis lyra & 0,01 & 0,08 & 1 & 1 \\
\hline 35 & Potamotrygon cf. orbignyi & 0,01 & 0,08 & 1 & 2 \\
\hline 36 & Serrabrycon magoi & 0,01 & 0,08 & 1 & 1 \\
\hline
\end{tabular}

Las especies destacadas con asterisco corresponden a las "especies objeto".

La frecuencia máxima hace referencia al número de lances por sitio.

De las 36 especies capturadas en caño Negro 33 correspondieron a especies incidentales, que representan el $92 \%$ de la pesca. En relación con los valores de abundancia, las especies objeto presentan un porcentaje de abundancia del $82 \%$ (Tabla 2).

\section{Río Orinoco afloramientos rocosos}

En la jornada de pesca llevada a cabo en el río Orinoco las especies objeto fueron dos: Hemiancistrus sp. y Peckoltia sp. 2. En este caso se pudo apreciar que la abundancia relativa de Hemiancistrus sp. $(0,44)$ es baja, pero su frecuencia es alta (23), mientras que para Peckoltia sp. 2 los valores de abundancia $(0,5)$ y frecuencia son bajos (2). Dentro de las capturas incidentales se observó que Aphanothorulus cf. ammophilus presenta los valores de abundancia $(0,9) \mathrm{y}$ frecuencia (25) más altos, seguido de Moenkhausia lepidura, con abundancia de 0,86 y frecuencia de 10 (Tabla 4).

Tabla 4. Valores de abundancia relativa en términos de CPUE $\left(n_{i}=c_{i} / t\right)$, porcentaje $(\%)$, frecuencia $(F)$ y número de individuos $(\mathrm{N})$, para las especies objeto e incidentales, colectadas en el río Orinoco (Afloramientos rocosos).

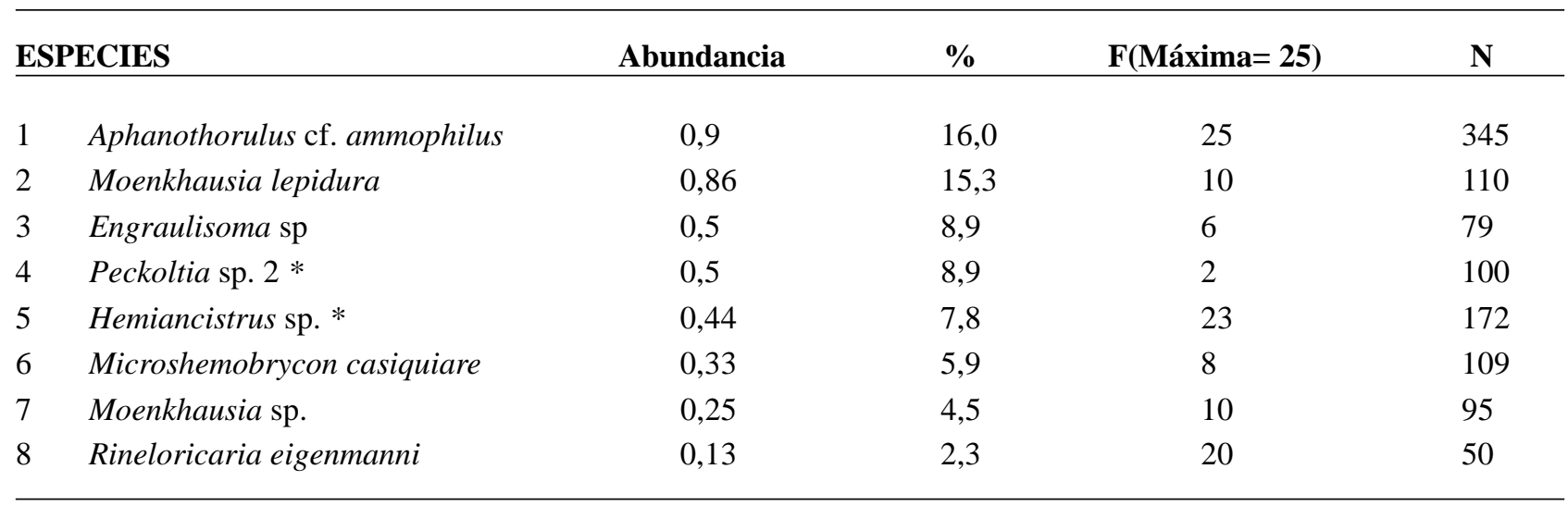




\begin{tabular}{|c|c|c|c|c|c|}
\hline \multicolumn{2}{|c|}{ ESPECIES } & \multirow{2}{*}{$\begin{array}{c}\text { Abundancia } \\
0,12\end{array}$} & \multirow{2}{*}{$\frac{\%}{2,1}$} & \multirow{2}{*}{$\begin{array}{c}\mathbf{F}(\text { Máxima }=\mathbf{~ 2 5}) \\
13\end{array}$} & \multirow{2}{*}{$\begin{array}{l}\mathbf{N} \\
46\end{array}$} \\
\hline 9 & Pseudolithoxus tigris & & & & \\
\hline 10 & Bryconamericus sp. & 0,09 & 1,6 & 10 & 33 \\
\hline 11 & Hypancistrus sp. & 0,09 & 1,6 & 10 & 33 \\
\hline 12 & Pseudoancistrus orinoco & 0,07 & 1,2 & 11 & 26 \\
\hline 13 & Hypancistrus sp. 2 & 0,06 & 1,1 & 3 & 8 \\
\hline 14 & Characidium sp. & 0,05 & 0,9 & 7 & 20 \\
\hline 15 & Creagrutus phasma & 0,05 & 0,9 & 9 & 20 \\
\hline 16 & Crenicichla geayi & 0,05 & 0,9 & 7 & 20 \\
\hline 17 & Ctenobrycon spilurus & 0,05 & 0,9 & 3 & 7 \\
\hline 18 & Hypostomus sp. & 0,05 & 0,9 & 2 & 7 \\
\hline 19 & Myloplus rubripinnis & 0,05 & 0,9 & 1 & 12 \\
\hline 20 & Aphyocharax alburnus & 0,04 & 0,7 & 6 & 15 \\
\hline 21 & Hypostomus cf. plecostomus & 0,04 & 0,7 & 2 & 6 \\
\hline 22 & Leporinus cf. aripuanaensis & 0,04 & 0,7 & 1 & 1 \\
\hline 23 & Satanoperca sp. & 0,04 & 0,7 & 9 & 16 \\
\hline 24 & Chilodus punctatus & 0,03 & 0,5 & 2 & 4 \\
\hline 25 & Lasciancistrus tentaculatus & 0,03 & 0,5 & 1 & 4 \\
\hline 26 & Leporellus vittatus & 0,03 & 0,5 & 3 & 8 \\
\hline 27 & Leporinus fasciatus & 0,03 & 0,5 & 1 & 1 \\
\hline 28 & Moenkhausia collettii & 0,03 & 0,5 & 1 & 4 \\
\hline 29 & Pachyurus schomburgkii & 0,03 & 0,5 & 4 & 10 \\
\hline 30 & Peckoltia sp. 3 & 0,03 & 0,5 & 8 & 103 \\
\hline 31 & Pimelodella sp. 2 & 0,03 & 0,5 & 2 & 4 \\
\hline 32 & Gnathodolus bidens & 0,02 & 0,4 & 3 & 6 \\
\hline 33 & Hemiancistrus sp. 1 & 0,02 & 0,4 & 4 & 8 \\
\hline 34 & Imparfinis sp. & 0,02 & 0,4 & 2 & 3 \\
\hline 35 & Plagioscion squamossisimus & 0,02 & 0,4 & 2 & 4 \\
\hline 36 & Roeboides affinis & 0,02 & 0,4 & 2 & 3 \\
\hline 37 & Vandellia becarii & 0,02 & 0,4 & 3 & 6 \\
\hline 38 & Anchoviella guianensis & 0,01 & 0,2 & 1 & 1 \\
\hline 39 & Ancistrus macrophthalmus & 0,01 & 0,2 & 1 & 1 \\
\hline 40 & Ancistrus trirradiatus & 0,01 & 0,2 & 1 & 1 \\
\hline 41 & Apareidon sp. & 0,01 & 0,2 & 1 & 1 \\
\hline 42 & Argonectes longiceps & 0,01 & 0,2 & 1 & 1 \\
\hline 43 & Brycon pesu & 0,01 & 0,2 & 1 & 2 \\
\hline 44 & Bryconamericus cismontanus & 0,01 & 0,2 & 2 & 2 \\
\hline 45 & Bryconamericus diaphanus & 0,01 & 0,2 & 2 & 2 \\
\hline 46 & Bryconops alburnoides & 0,01 & 0,2 & 3 & 3 \\
\hline 47 & Bryconops caudomaculatus & 0,01 & 0,2 & 1 & 1 \\
\hline 48 & Cichla sp. & 0,01 & 0,2 & 2 & 3 \\
\hline 49 & Crenicichla sp. 5 & 0,01 & 0,2 & 1 & 1 \\
\hline 50 & Eigenmannia virescens & 0,01 & 0,2 & 4 & 4 \\
\hline 51 & Exodon parodoxus & 0,01 & 0,2 & 1 & 1 \\
\hline
\end{tabular}




\begin{tabular}{|c|c|c|c|c|c|}
\hline \multicolumn{2}{|c|}{ ESPECIES } & \multirow{2}{*}{$\begin{array}{c}\text { Abundancia } \\
0,01\end{array}$} & \multirow{2}{*}{$\frac{\%}{0,2}$} & \multirow{2}{*}{ F(Máxima= 25) } & \multirow{2}{*}{$\begin{array}{l}\mathbf{N} \\
2\end{array}$} \\
\hline 52 & Farlowella sp. & & & & \\
\hline 53 & Hassar orestis & 0,01 & 0,2 & 2 & 5 \\
\hline 54 & Hydrolycus scomberoides & 0,01 & 0,2 & 1 & 1 \\
\hline 55 & Hypoptopoma steindachneri & 0,01 & 0,2 & 2 & 3 \\
\hline 56 & Hypostomus sp. 2 & 0,01 & 0,2 & 1 & 1 \\
\hline 57 & Lamontichthys cf. llanero & 0,01 & 0,2 & 1 & 1 \\
\hline 58 & Leporinus agassizii & 0,01 & 0,2 & 6 & 10 \\
\hline 59 & Leporinus cf. brunneus & 0,01 & 0,2 & 2 & 5 \\
\hline 60 & Leporinus friderici & 0,01 & 0,2 & 2 & 2 \\
\hline 61 & Loricariichtys cf. brunneus & 0,01 & 0,2 & 1 & 1 \\
\hline 62 & Microglanis iheringi & 0,01 & 0,2 & 1 & 1 \\
\hline 63 & Moenkhausia cf. miangi & 0,01 & 0,2 & 1 & 1 \\
\hline 64 & Moenkhausia grandisquamis & 0,01 & 0,2 & 1 & 1 \\
\hline 65 & Moenkhausia megalops & 0,01 & 0,2 & 1 & 2 \\
\hline 66 & Ochmacanthus alternus & 0,01 & 0,2 & 1 & 1 \\
\hline 67 & Peckoltia sabaji & 0,01 & 0,2 & 1 & 1 \\
\hline 68 & Peckoltia sp. 1 & 0,01 & 0,2 & 3 & 5 \\
\hline 69 & Phenacogaster cf. microstictus & 0,01 & 0,2 & 2 & 3 \\
\hline 70 & Pimelodella cristata & 0,01 & 0,2 & 3 & 5 \\
\hline 71 & Pimelodella limani & 0,01 & 0,2 & 2 & 3 \\
\hline 72 & Pimelodella metae & 0,01 & 0,2 & 1 & 1 \\
\hline 73 & Pimelodella sp. 1 & 0,01 & 0,2 & 1 & 2 \\
\hline 74 & Pimelodella sp. 3 & 0,01 & 0,2 & 1 & 1 \\
\hline 75 & Pimelodus blochi & 0,01 & 0,2 & 4 & 5 \\
\hline 76 & Pimelodus ornatus & 0,01 & 0,2 & 1 & 1 \\
\hline 77 & Schultzichthys bondi & 0,01 & 0,2 & 1 & 1 \\
\hline 78 & Semaprochilodus laticeps & 0,01 & 0,2 & 1 & 1 \\
\hline 79 & Serrasalmus irritans & 0,01 & 0,2 & 1 & 1 \\
\hline 80 & Tetragonopterus argenteus & 0,01 & 0,2 & 1 & 2 \\
\hline 81 & Thoracocharax stellatus & 0,01 & 0,2 & 1 & 1 \\
\hline 82 & Triportheus sp. 1 & 0,01 & 0,2 & 1 & 1 \\
\hline
\end{tabular}

Las especies destacadas con asterisco corresponden a las "especies objeto".

La frecuencia máxima hace referencia al número de lances por sitio.

De las 82 especies capturadas en los afloramientos rocosos $80(98 \%)$ correspondieron a incidentales. En relación con los valores de abundancia, las especies objeto presentaron un porcentaje del 17\% (Tabla 2).

\section{Río Orinoco, pozo Bisal}

En el muestreo llevado a cabo en el pozo Bisal, la especie objeto fue Corydoras cf. melanistius. Esta presenta valo- res de abundancia relativa $(17,07)$ y frecuencia $(\mathrm{F}=2)$ más altos, mientras que para las incidentales, estos valores corresponden a los de Triportheus angulatus (Tabla 5).

De las 28 especies capturadas en el pozo Bisal 27 correspondieron a incidentales, y representan el $96 \%$ de la pesca. En relación con los valores de abundancia, la especie objeto presentó un porcentaje de abundancia del 83\% (Tabla 2). 
Tabla 5. Valores de abundancia relativa en términos de CPUE $\left(n_{i}=c_{i} / t\right)$, porcentaje $(\%)$, frecuencia $(F)$ y número de individuos $(\mathrm{N})$, para las especies objeto e incidentales, colectadas en el río Orinoco (pozo Bisal).

\begin{tabular}{|c|c|c|c|c|c|}
\hline \multicolumn{2}{|c|}{ ESPECIES } & \multirow{2}{*}{$\begin{array}{c}\text { Abundancia } \\
17,07\end{array}$} & \multirow{2}{*}{$\frac{\%}{83,39}$} & \multirow{2}{*}{$\frac{\text { F (Máxima= 2) }}{2}$} & \multirow{2}{*}{$\frac{\mathbf{N}}{107}$} \\
\hline 1 & Corydoras cf. melanistius* & & & & \\
\hline 2 & Triportheus angulatus & 1,25 & 6,11 & 2 & 89 \\
\hline 3 & Aequidens tetramerus & 0,77 & 3,76 & 2 & 55 \\
\hline 4 & Psectrogaster ciliata & 0,36 & 1,76 & 2 & 51 \\
\hline 5 & Curimatella dorsalis & 0,3 & 1,47 & 2 & 42 \\
\hline 6 & Gymnocorymbus bondi & 0,09 & 0,44 & 2 & 3 \\
\hline 7 & Callichthys callichthys & 0,07 & 0,34 & 1 & 5 \\
\hline 8 & Hoplias malabaricus & 0,07 & 0,34 & 2 & 10 \\
\hline 9 & Crenicichla sp. 3 & 0,06 & 0,29 & 1 & 1 \\
\hline 10 & Hypostomus sp. & 0,06 & 0,29 & 1 & 4 \\
\hline 11 & Hoplerythrinus unitaeniatus & 0,05 & 0,24 & 2 & 7 \\
\hline 12 & Semaprochilodus laticeps & 0,05 & 0,24 & 2 & 7 \\
\hline 13 & Acestrorhynchus microlepis & 0,03 & 0,15 & 2 & 2 \\
\hline 14 & Aequidens chimantanus & 0,03 & 0,15 & 2 & 2 \\
\hline 15 & Ancistrus trirradiatus & 0,03 & 0,15 & 2 & 2 \\
\hline 16 & Chaetobranchus flavescens & 0,03 & 0,15 & 2 & 2 \\
\hline 17 & Glyptoperichtys gibbiceps & 0,03 & 0,15 & 2 & 2 \\
\hline 18 & Brycon cf. melanopterus & 0,02 & 0,10 & 2 & 3 \\
\hline 19 & Brachyhyроротиs cf. brevirostris & 0,01 & 0,05 & 1 & 1 \\
\hline 20 & Crenicichla sp. 1 & 0,01 & 0,05 & 2 & 4 \\
\hline 21 & Crenicichla sp. 4 & 0,01 & 0,05 & 1 & 1 \\
\hline 22 & Electrophorus electricus & 0,01 & 0,05 & 1 & 1 \\
\hline 23 & Goeldiella eques & 0,01 & 0,05 & 1 & 1 \\
\hline 24 & Leporinus cf. agassizii & 0,01 & 0,05 & 1 & 1 \\
\hline 25 & Mesonauta egregius & 0,01 & 0,05 & 1 & 1 \\
\hline 26 & Platydoras costatus & 0,01 & 0,05 & 1 & 1 \\
\hline 27 & Satanoperca sp. & 0,01 & 0,05 & 1 & 1 \\
\hline 28 & Serrasalmus irritans & 0,01 & 0,05 & 1 & 1 \\
\hline
\end{tabular}

Las especies destacadas con asterisco corresponden a las "especies objeto".

La frecuencia máxima hace referencia al número de lances por sitio.

\section{Discusión}

\section{Descripción de la pesca ornamental en el área de influencia del municipio de Puerto Carreño}

La pesca de especies de interés ornamental se lleva a cabo en los principales caños, lagunas y ríos de la zona de influencia del municipio de Puerto Carreño. Las especies objeto de cada jornada dependen de los requerimientos de los centros de acopio, es decir depende de los encargos que se hacen desde las diferentes ciudades; por esta razón, algunas especies para unas jornadas son objeto y para otras son incidentales. Caso contrario a lo que sucede en Venezuela, allí, los pescadores salen a sus faenas colectando todo lo capturado, no se seleccionan las especies, y todas estas son acopiadas esperando los encargos de las diferentes ciudades (com. per. Provenzano). Los ríos Meta, Orinoco, Bita y Tomo son los lugares de extracción más importantes de las diferentes especies. Los afluentes de estos ríos como por ejemplo caño Negro también son puntos de extracción 
importantes. El arte de pesca depende de la especie de interés y del lugar de extracción. Para la captura de especies de variedad, en su mayoría Characiformes, se utiliza la red de arrastre o "chinchorro", la cual es poco selectiva y permite que se capturen diversas especies que no son objeto en la pesca, las que son dejadas en la orilla o en algunos de los casos son devueltas al río por los pescadores. Durante las faenas, se recorren las orillas para poder divisar los cardúmenes, una vez son vistos se efectúan los lances; aún así, esto no garantiza que se capture la especie objeto en alto número. Para la pesca de especies que no forman grandes cardúmenes se hace uso de la nasa (arawana, Osteoglossum ferreirai) o del rayero (rayas, Potamotrygon spp), este tipo de pesca es especifico y no capturan especies incidentales. La pesca de Loricaridos se efectúa en afloramientos rocosos, para lo cual se utiliza la careta y/o la red de arrastre, esta última es similar a la usada en la pesca de variedad pero en la parte inferior lleva un plomo más pesado. El uso de la careta permite al pescador hacer varias inmersiones para colectar uno a uno los ejemplares requeridos. El uso de la red de arrastre es poco selectivo, se efectúan múltiples lances y arrastres. A diferencia de la pesca de variedad, y debido al comportamiento de las especies de cuchas, el pescador no divisa los cardúmenes antes de efectuar el lance. Debido a lo anterior, el número de especies incidentales es mayor con respecto al de otras especies.

\section{Riqueza}

En total fueron colectadas 152 especies. Este valor es menor al registrado previamente para el Río Tomo (7) con 282 especies y para la cuenca del Orinoco con 995 especies (8). Maldonado-Ocampo (9) presenta un listado de 52 especies para Puerto Carreño. Tal diferencia se atribuye a los diferentes objetivos propuestos en cada trabajo; estos últimos dirigidos a inventarios ictiofaunisticos y el presente a evaluar las capturas incidentales de la pesca ornamental.

Por otro lado, a los listados presentados en las investigaciones anteriores, se suma el trabajo de Galvis et al., 2007 (10) quienes registran un total de 301 especies de interés ornamental de la Orinoquia Colombiana; algunas de estas especies fueron colectadas en centros de acopio en diferentes ciudades. De acuerdo a esto, un aumento en el área de muestreo y en el esfuerzo de pesca para los diferentes periodos del año, podrían incrementar el número de especies, para la zona de influencia del municipio de Puerto Carreño.

Lasso et al., 2004 (8), presentan un listado de las especies reportadas para la cuenca del Orinoco, dividido por subcuencas. Sin embargo, Asterophysus batrachus apare- ce reportada para Atabapo, Apure y Caura, pero no para la zona de influencia del Municipio de Puerto Carreño, Colombia. La "cucha Diamante (Hemiancistrus sp.), no está registrada en el trabajo de Galvis et al., 2007 (10), quienes publican un listado de las especies de interés ornamental para la Orinoquia colombiana. Es así como la presente investigación contribuye con información para dos nuevas especies en el área de estudio.

En general, los Characiformes son el grupo dominante con 74 especies, seguido de los Siluriformes con 47. Las características de la pesquería ornamental, en donde se practica principalmente de día, así como las artes empleadas explican el por qué se seleccionan las capturas hacia los Characiformes. Además, se debe considerar que este orden es el que se reporta más abundante y frecuente para la Orinoquia. Por otro parte, el número considerable de especies de Siluriformes puede deberse a que algunas de estas fueron especies objeto durante las faenas, por lo que la pesca se llevaba a cabo en afloramientos rocosos, ambientes en donde son fáciles de encontrar y capturar.

\section{Abundancia relativa, frecuencia y porcentaje de especies objeto vs. incidentales.}

\section{Río Bita}

Según la tabla 1, Hemigrammus sp., Moenkhausia sp., Bryconamericus sp., y Carnegiella marthae, presentan abundancias de captura relativamente similares, variando entre 2.0 y 2.2. Sin embargo, para estas especies la frecuencia presentó valores diferentes; así por ejemplo, Hemigrammus sp. presentó una alta frecuencia (8) en tanto que para, Carnegiella marthae, su frecuencia es baja ya que solo fue colectada en un lance. Es decir, si Carnegiella marthae, fuese una especie de interés durante alguna de las faenas de pesca, se necesitaría de un esfuerzo y un tiempo de pesca menor para alcanzar una cifra alta de captura total. Si esta cifra de captura se obtiene a partir de un menor esfuerzo y un menor tiempo efectivo de pesca, en este caso, el resultado de la pesca podría considerarse como exitoso (5). La pesca de Carnegiella marthae, se realiza divisando por las orillas los cardúmenes y luego se efectúa el lance y el arrastre, por lo que el número de individuos y de especies incidentales resulta ser bajo.

\section{Caño Negro}

En este sitio Corydoras cf. melanistius fue la especie objeto más abundante (> 43\%) y presentó la máxima frecuencia (8/8). Estos altos valores se explican a partir de la técnica 
de pesca. Durante la época de aguas bajas, se forman resacas, es decir, cuerpos de agua de la zona riparia que quedan aislados del cauce principal, dentro de los cuales permanecen atrapadas muchas especies, por lo que su captura es fácil de realizar, con artes como la red de arrastre.

Las capturas efectuadas en el cauce principal de caño Negro, muestran un comportamiento muy parecido a lo observado en el río Bita puesto que las técnicas y los artes de pesca son muy similares. Así, Dicrossus maculatus, al igual que en el río Bita, fue la especie objeto más abundante y más frecuente. En la pesca incidental, Moenkhausia, Hemigrammus y Nannostomus, constituyen los grupos más abundantes y frecuentes; resultados similar a lo observado en el río Bita, con Hemigrammus, Bryconamericus y Moenkhausia. Estos resultados muestran que la pesca de variedad efectuada por los pescadores, a pesar de no ser una técnica estandarizada, puede ser utilizada para comparar los datos obtenidos en dos sitios diferentes. Las abundancias relativas para los grupos de pesca incidental del río Bita, son altos comparados con los valores estimados para caño Negro, lo cual puede deberse a que el tiempo efectivo de pesca y la duración de las jornadas fue mayor para el Bita.

\section{Afloramientos rocosos, río Orinoco}

En la pesca efectuada en los afloramientos rocosos del río Orinoco, las especies de interés corresponden a individuos de la familia Loricariidae. Estos afloramientos rocosos se presentan a lo largo del cauce principal del Río Orinoco. Al considerar el Río Orinoco como un sistema abierto y extenso, el esfuerzo de muestreo resulta ser pequeño con relación a aquel, por lo que es válido suponer que las relaciones lineales entre la captura y el esfuerzo no se alteran (5). Es decir, en sistemas abiertos, con alta inmigraciónemigración de peces y, quizá, con especies no territoriales (5), y a pesar de que las colectas se realizan siempre en los mismos afloramientos rocosos durante varios días, se considera que lo colectado hoy no afectará lo colectado mañana. Es así como los valores de CPUE y de frecuencia de ocurrencia obtenidos para este sitio de extracción, no se ven afectados por la extracción de individuos en los mismos sitios a lo largo de toda la jornada.

La segunda jornada en los afloramientos rocosos en el Río Orinoco, permite observar un cambio en la composición de especies incidentales. En este caso, los géneros Moenkhausia y Hemigrammus resultaron ser poco abundantes. Esto puede deberse a que las comunidades de peces continentales exhiben cambios durante el ciclo anual. Cuando se realizo la segunda jornada en los afloramientos rocosos, los niveles del río Orinoco se habían incrementado considerablemente, entre 10 a $15 \mathrm{~m}$ con relación a la primera. Los periodos hidrológicos definen los fenómenos de subienda y bajanza, por lo anterior, las capturas y las capturas por unidad de esfuerzo podrían cambiar ampliamente de un mes a otro.

\section{Pozo Bisal, río Orinoco}

La pesca efectuada en la resaca "pozo Bisal", presentó resultados similares a los obtenidos en la pesca de Corydoras cf. melanistius efectuada en caño Negro. Esta especie fue objeto en las dos jornadas, presentando abundancia y frecuencia altas. Esto se explica a partir de la técnica de pesca en donde se extiende la red sobre una resaca y se efectúa el arrastre colectando así la mayor parte de los individuos allí presentes.

Por último, a cada población y tipo de arte debe corresponder una CPUE distinta, por lo que es común obtener índices diferentes de CPUE para cada especie o grupo de especies, flotilla y temporada (5). Sin embargo, se puede apreciar, que a pesar de haber efectuado los muestreos en diferentes lugares, géneros como Moenkhausia y Hemigrammus hacen parte regular de las colectas incidentales en los tres sitios, a excepción de la pesca en las resacas. Estos dos géneros, son generalista, por lo que se encuentran en una gran diversidad de ambientes, lo cual explica la alta frecuencia de ocurrencia que presentaron en las colectas en los diferentes sitios.

\section{Historia de vida y hábitos alimentarios especies ícticas más frecuentes y abundantes.}

Las poblaciones naturales están comprendidas por organismos finitos y por eso están sujetas a una renovación de individuos gradual o periódica (11). La estructura de edad y el tamaño de una población son resultados de la natalidad y la mortalidad, las cuales están definidas por la "historia de vida" (11). Así, para algunas especies de las cuales se cuenta con información, los resultados de abundancia se interpretan a partir de dichas historias de vida, considerando tanto las estrategias reproductivas, como los hábitos alimentarios de las especies objeto e incidentales.

Dicrossus maculatus: Esta especie presenta estrategia de reproducción " $k$ " por lo que la densidad poblacional es estable y la sobrevivencia de adultos y jóvenes es relativamente alta (12), lo anterior favorece que sea una especie abundante y frecuente 
Hemigrammus: Son especie omnívoras, se alimentan de semillas, microcrustáceos e insectos, su fecundidad es de aproximadamente 400-500 huevos por desove y presenta estrategia reproductiva $\mathrm{r} 1$ (13). A partir de lo anterior se puede apreciar que las especies de este género, al ser omnívoras y al aprovechar, además, toda la columna de agua para alimentarse, conlleva a que su frecuencia y abundancia sean altas. Por otro lado, al ser especies con estrategia r1 presentan iteroparidad y ciclos de vida cortos por lo que son especies abundantes en el hábitat.

Corydoras cf. melanistius: Esta especie presentan estrategia reproductiva " $\mathrm{r} 2$ " (12), las especies $\mathrm{r} 2$ presentan alta sobrevivencia de adultos, alta fecundidad, y grandes fluctuaciones en la densidad poblacional (11). Además, en campo se observó esta especie subir a la superficie del agua, probablemente con la finalidad de tomar aire atmosférico y realizar respiración aérea facultativa. La captura de esta especie se llevó a cabo en resacas, las cuales son cuerpos de agua estacional que presentan alta turbidez y temperatura y oxígeno disuelto bajo. Así, las características de la especie le permiten vivir bajo las condiciones de estos hábitats .

\section{Conclusiones}

Durante las diferentes jornadas de pesca se colectaron 152 especies; los grupos con mayor número de especies son Characiformes con 74, Siluriformes con 47 y Perciformes con 23. Le siguen Gymnotiformes (3), Rajiformes (2) y Clupeiformes, Beloniformes y Osteoglossiformes con una especie cada uno. El gran número de especies del orden Characiformes, se debe a que este orden es el más abundante y frecuente para la región de la Orinoquia; además, las diferentes faenas de pesca se realizaron en las horas del día, momento donde están más activos. Por otro lado, el comportamiento particular de cada especie se refleja en las capturas; por ejemplo, Potamotrygon motoro, especie que no forma cardúmenes pero aparentemente territorialista, presentó baja abundancia pero frecuencia alta; por el contrario, Carnegiella marthae que tiene un comportamiento gregario y migratorio, presento alta abundancia pero baja frecuencia.

A partir del estudio se pudo determinar que las especies objeto de la pesca ornamental también pueden ser, en otras circunstancias, especies incidentales. De forma semejante, las incidentales también pueden llegar a ser objeto. Lo que las define como tal es si en la jornada específica de pesca son objetivo o no de esta. La pesca ornamental básicamente se realiza por encargo, es decir por demanda y es escasa la cultura del acopio de especies que en ese momento no tienen pedido.
Las capturas ícticas incidentales, dependen de la especie ornamental de interés, de la técnica y el arte de pesca. De este modo, especies capturadas con artes de pesca altamente selectivos, como los utilizados en la pesca de la Arawana (Osteoglossum ferreirai) y la raya motora (Potamotrygon motoro), no presentan capturas ícticas incidentales, lo cual indica, que la pesca de estas especies no afecta a otras. Por otro lado, el uso de artes de pesca poco selectivos, como la red de arrastre o chinchorro, en la captura de Corydoras cf. melanistius, Dicrossus maculatus y Hemiancistrus sp., presentan un gran porcentaje de especies ícticas incidentales. Además, el impacto de la pesca de especies ornamentales sobre las especies incidentales se puede apreciar a nivel de número de especies (riqueza); sin embargo, los valores de abundancia estimados a partir de la captura por unidad de esfuerzo (CPUE) frecuentemente fueron menores para estas especies incidentales que para las especies objetivo de la pesca. La excepción se observó en los afloramientos rocosos del río Orinoco en donde el impacto se apreció tanto en la riqueza como en la abundancia de las especies incidentales.

El presente trabajo, registra dos nuevas especies para el área de estudio: el Tongolino (Asterophysus batrachus) y la cucha diamante (Hemiancistrus sp.).

\section{Agradecimientos}

A Francisco Provenzano, Donald Taphorn y Armando Ortega por su colaboración en la identificación taxonómica de las especies; a los pescadores de Punta de Laja, municipio de Puerto Carreño. Los revisores del presente artículo realizaron valiosos aportes.

\section{Financiación}

Esta investigación se realizó dentro del marco del Proyecto: "Incremento y consolidación de áreas protegidas y generación de alternativas económicas sostenibles enfocadas en la pesca ornamental y el ecoturismo en la Reserva de Biosfera el Tuparro a través de un esquema participativo", convenio de Asignación de Donación Unión Temporal Omacha Verde y El Fondo Para la Acción Ambiental y la Ninez. La Pontificia Universidad Javeriana - sede Bogotá, también participó como cofinanciadora.

\section{Conflicto de intereses}

Los estudios que se han realizado para la descripción de la pesca ornamental, sólo permiten tener un conocimiento par- 
cial de éste. Algunos de estos estudios han generado una lista provisional de especies ornamentales, que año tras año se ha venido actualizando, dando como resultado un registro de especies cada vez más completo. Sin embargo, para la pesca ornamental no se han realizado estudios de las capturas incidentales. Debido a lo anterior, no se conoce la magnitud del impacto que causa la pesca de especies ornamentales en la comunidad íctica y en la economía de la región. Siendo así, es necesario realizar monitoreos de las capturas ícticas incidentales. Por esta razón es importante que a partir de esta investigación, se lleven a cabo más estudios a nivel de biología y dinámica poblacional que permitan estimar el impacto real de la pesca ornamental sobre las especies objetivo e incidentales.

\section{Referencias}

1. Ramírez-Gil H., L, Carrillo, E. Lacera y R, Ajiaco. La Pesca De Especies De Interés Ornamental En El Área De Influencia De Puerto Carreño. En: Ramírez-Gil H. y Ajiaco-Martínez R.H. La pesca en la Baja Orinoquia colombiana: pasado, presente y futuro Instituto Nacional De Pesca y Acuicultura, INPA. Colombia, 2001.

2. Mancera-Rodríguez. J, L. Álvarez-León. Comercio de peces ornamentales en Colombia. Acta biológica. Colombiana., 2008; 13 (1): 23 - 52

3. Sistema de información de Pesca y Acuicultura. Comportamiento de las capturas de peces ornamentales años 2006-2007. Boletín mensual mayo de 2008.

4. Ramírez-Gil H y R. Ajiaco. La Orinoquia Colombiana y Su Área De Frontera. En: La Pesca En La Baja Orinoquia Colombiana: Una Visión Integral. Instituto Nacional De Pesca y Acuicultura, INPA. Colombia, 2001.

5. FAO, Organización de las naciones unidas para la agricultura y la alimentación. Directrices para la recopilación sistemática de datos relativos a la pesca de captura. Roma, 1998.
6. Hued, A. C., M. Bistoni. Abundancia Y Distribución De Las Especies Ícticas (Osteichthyes) Del Río San Francisco-Cosquín, Córdoba, Argentina. Iheringia, Sér. Zool. Porto Alegre 2001, 27 (91): 75-78

7. Maldonado-Ocampo. J, Lugo M., Bogotá-Gregory J.D., Lasso C.A., Vásquez L., Usma J.L., Taphorn D.C., F. Provenzano. Peces del río Tomo, cuenca del Orinoco, Colombia. Biota Colombiana 2006, 7(1): 113-128

8. Lasso C.A., Mojica J.I., Usma J.S., Maldonado-O. J.A., DoNascimento C., Taphorn D.C., Provenzano F., LassoAlcalá O.M., Galvis G., Vásquez L., Lugo M., Machado-Allison A., Royero R., Suárez C., A. Ortega-Lara. Peces de la cuenca del río Orinoco. Parte I: lista de especies y distribución por subcuencas. Biota Colombiana 2004; 5(2): 95-158

9. Maldonado, J. Peces de Puerto Carreño. Lista Ilustrada. Fundación Omacha. Colombia, 2000.

10. Galvis, G., J. I. Mojica, F. Provenzano, C. A. Lasso, D. C. Taphorn, R. Royero, C. Castellanos Castillo, A. Gutierrez, Y. López, L. M. Mesa, P. Sanchez, C. A. Cipamocha. Peces de la Orinoquia colombiana con énfasis en especies de interés ornamental. INCODER; Universidad Nacional de Colombia. Bogotá D.C, Colombia, 2007.

11. Winemiller, O. K.; Taphorn, D. C. La evolución de las estrategias de vida en los peces de los llanos occidentales de Venezuela. Biollania 1989 6: 77-122.

12. Froese, R. and D. Pauly. Editors. 2008.FishBase. World Wide Web electronic publication. http:// www. fishbase. org, consultado el 30 de enero de 2010

13. Taphorn, D. C. The characiform fishes of the Apure river drainage, Venezuela. Biollania. Edición especial 4. 537 p. 1992. 